\title{
Association of Glutathione S-Transferase Polymorphisms with Dietary Composition but not Anthropometry in Obese as well as Non-Obese Individuals
}

Barbara Klánová ${ }^{1}$, Filip Zlámal ${ }^{2}$, Aneta Pohořalá ${ }^{3}$, Ondrej Slaby ${ }^{4-5}$, Hynek Pikhart ${ }^{6}$, Julie BienertováVaškü ${ }^{2}$

${ }^{1}$ Department of Neonatology, Center for Reproductive Medicine, University Hospital Brno, Obilní trh 11, Brno 602 00, Czech Republic

${ }^{2}$ Research Centre for Toxic Compounds in the Environment (RECETOX), Faculty of Science, Masaryk University, Kamenice 5, A29, 625 00, Brno, Czech Republic

${ }^{3}$ Department of Preventive Medicine, Faculty of Medicine, Masaryk University, Kamenice 5 A21, Brno 625 00, Czech Republic

${ }^{4}$ Central European Institute of Technology, Masaryk University, Brno, Czech Republic

${ }^{5}$ Department of Comprehensive Cancer Care, Masaryk Memorial Cancer Institute, Faculty of Medicine, Masaryk University, Brno, Czech Republic

${ }^{6}$ Research Department of Epidemiology and Public Health, University College London, 1-19 Torrington Place, London WC1E 6BT, United Kingdom

*Address for correspondence:

Assoc. Prof. Julie Bienertová-Vašků, MD, Ph.D.

Research Centre for Toxic Compounds in the Environment (RECETOX), Faculty of Science, Masaryk University, Kamenice 5, A29, 625 00, Brno, Czech Republic

Phone: +420549498439

Fax: +420549494340

E-mail: jbienert@med.muni.cz 


\section{Abstract}

\section{Objectives}

Glutathione S-transferases (GSTs) are detoxifying enzymes for a number of substrates including some food compounds. Selected GST polymorphisms have been proven to significantly affect enzymatic activity; however, it is unclear whether this altered metabolism influences dietary composition. The objective of this study was to locate the correlation between GST polymorphisms and selected nutritional parameters, namely fiber and vitamin C intake.

\section{Methods}

This study was conducted on a cohort of 472 individuals (mean age 45.26; mean BMI 32.36) from the South Moravian region of the Czech Republic. Basic anthropometrical parameters were measured and no association was found for the selected polymorphisms. Polymorphisms in GSTA1, GSTM1 and GSTT1 were genotyped using a PCR-based methodology. Food intake was monitored using a selfadministered 7-day questionnaire which was subsequently analyzed with special focus on vitamin C intake, fiber intake and total energy intake.

\section{Results}

For GSTA1 and GSTM1 polymorphisms, an association was observed with fiber intake. While no association was found with vitamin $C$ intake, mean vitamin $C$ intake was found to be higher than recommended daily value. No association was found with either daily energy intake or anthropometric parameters.

\section{Conclusion}

Based on our results, GST polymorphisms seem to affect dietary composition; however, they have no effect on total energy intake or any association with obesity.

Key words

Glutathione S-Transferase, Genetic polymorphisms, Dietary composition, Food records 


\section{Background}

Diet and nutrition are crucial factors in health improvement and preservation. Vegetables are an essential part of a balanced diet and provide a source of fiber and a number of nutrients, namely vitamins $\mathrm{C}, \mathrm{A}$ and $\mathrm{K}$, folate and potassium. According to the World Health Organization, eating at least 400-500 g, or 5 portions, of fruit and vegetables per day reduces the risk of non-communicable diseases (such as diabetes, heart disease, stroke and cancer) and helps ensure an adequate daily intake of dietary fiber (1). Adequate fiber intake is $14 \mathrm{~g}$ per 1,000 calories, or $25 \mathrm{~g}$ per day for women and $38 \mathrm{~g}$ for men (2). The recommended nutrient intake for vitamin C for adults is $45 \mathrm{mg}$ per day (3). In data published by the European Food Safety Society the mean consumption of fruit and vegetables is $386 \mathrm{~g}$ per day, varying between different countries (4).

Lower daily intake of vegetables can be explained by demographic and social factors as well as interindividual variations in the ability to metabolize different compounds in vegetables. This variability is believed to be affected by the environment, genetics and gut microflora (5). While the correlation between human metabolic phenotypes and specific dietary preferences has been described previously (6), no systematic studies focusing specifically on the correlation with vegetable intake are currently available. The association of genetics with the metabolism of various phytochemicals in fruits and vegetables has thus yet to be examined.

Conjugation with glutathione constitutes an important metabolic pathway for a number of phytochemicals in vegetables and fruit. Glutathione transferases (also known as glutathione Stransferases or GSTs) are a superfamily of enzymes which catalyze the conjugation of substrates to glutathione in phase II of the detoxification process, function as indicators of peroxidase and isomerase activity and protect against $\mathrm{H}_{2} \mathrm{O}_{2}$-induced cell death. In addition, they are capable of binding non-catalytically to a wide range of both endogenous and exogenous ligands (7). The three major families with glutathione transferase enzymatic activity are cytosolic, mitochondrial and microsomal GST. Cytosolic GST consists of 4 main classes: Alpha, Mu, Pi and Theta. Alpha and Mu are the major hepatic GSTs. GSTM1 and GSTT1 have been shown to conjugate isothiocyanates and indoles found especially in brassica vegetables (8). Isothiocyanates (ICNs) have been widely studied for their possible anticancerogenic effect $(9,10)$ which has been ascribed to their cytotoxicity. ICNs are major metabolic breakdown products of glucosinolates. In humans, glucosinolates are converted into ITCs by enteric microflora, and are subsequently metabolized by conjugation with glutathione and ultimately with N-acetyl-L-cysteine (11). However, GST also reacts with other phytochemicals such as diallyl disulphide (found in garlic), aflatoxins and possibly some compounds yet to be identified.

A number of studies describe mutations in glutathione S-transferase. The genes coding these enzymes are highly polymorphic and individual allelic variants arise from single nucleotide polymorphisms (SNPs) or multi-base deletions or rearrangements. Some mutations in GSTM1 and GSTT1 lead to the absence of the functional enzyme and are therefore directly associated with altered enzyme activity. Depending on factors including ethnicity, the GSTM1-null genotype occurs in 39 to $63 \%$ of the population while GSTT1-null is present in 10 to $64 \%$ (8). Several studies indicate that different polymorphisms in GST can affect the metabolism of ICNs and, by extension, other phytochemicals metabolized by GSTs. 
Even though GST polymorphisms have been studied several times in relation to cancer prevention, they have never been studied as a reason for the altered digestion of certain vegetables. The aim of this study is thus to investigate whether a correlation exists between polymorphisms in selected GSTs and the dietary composition of adults, specifically concentrating on the intake of fiber and vitamin $\mathrm{C}$ which may represent possible differences in vegetable intake. Identifying a relationship between these factors would help us understand the need for individualized adult diets.

\section{Methods}

\section{Study subjects}

The study subjects were Czech Caucasian individuals recruited for the present study in a mass media campaign addressing the population of the South Moravian region of the Czech Republic (12). The study group consisted of 472 individuals (mean BMI 32.36; BMI range 47.36; mean age 45.26; age range 57.40).

Data on personal or family history of obesity, birth weight, age at onset of obesity and its severity were obtained by a professional interviewer using a semi-structured questionnaire. A positive family history of obesity was estimated as having at least one obese relative with $\mathrm{BMI} \geq 30 \mathrm{~kg} / \mathrm{m} 2$ in the close family (siblings, parents and their siblings, or grandparents). Both the obese cases and the nonobese controls underwent the same examination focused on their anthropometric characteristics; the subjects of the study were also interviewed with respect to their current smoking status and its history. These anthropometric measurements included weight, height, BMI, lean body mass, fat mass, body fat, waist and hip circumference, waist:hip ratio and skinfold thickness. Body composition was measured by bioelectrical impedance analysis using a BodyStat Ltd bioimpedance analyzer with the subject lying in a supine position. Height was measured with a calibrated stadiometer and weight (in light indoor clothing and without shoes) was measured using a precisely calibrated set of scales (13).

Participants were asked to complete standardized 7-day food records. Data obtained from the study subjects were subsequently analyzed (13), with special attention devoted to total energy intake, dietary fiber intake and vitamin $\mathrm{C}$ intake. All procedures were carried out in accordance with ethical standards set out by the local ethical committee on human experimentation and with the Helsinki Declaration of 1975, as revised in 2000. Informed consent was obtained from all patients for being included in the study.

\section{DNA isolation and genotyping}

DNA isolation and genotyping was carried out as described previously by Hezova et al. (14). Genomic DNA was isolated from whole peripheral blood and DNA concentration was measured using a Nanodrop ND-1000. Realtime PCR allelic discrimination was carried out using a StepOne Real-Time PCR instrument. PCR-restriction fragment length polymorphism (RFLP) was used for analysis (15). PCR was performed with a total volume of $12.5 \mu \mathrm{l}$, thermocycler parameters included an initial five-minute denaturation step at $94^{\circ} \mathrm{C}$, then thirty cycles of denaturation, annealing and extension and a final seven-minute extension and cooling reaction to $4{ }^{\circ} \mathrm{C}$. Digestion was performed for 12 hours at $37^{\circ} \mathrm{C}$ and stopped at $65^{\circ} \mathrm{C}$ for 20 minutes. Digested products were isolated on $2 \%$ agarose gel stained with 
ethidium bromide. GSTM1 and GSTT1 deletions were genotyped using a duplex PCR with the albumin gene serving as an internal positive control. The total reaction volume was $12.5 \mu \mathrm{l}$. PCR conditions were the same as for the amplification of GSTA1. PCR products were isolated on $2 \%$ agarose gel stained with ethidium bromide. The wild-type genotype for GSTM1 corresponded to $215 \mathrm{bp}$, GSTT1 to $480 \mathrm{bp}$ and the internal control to $380 \mathrm{bp}(16,17)$.

\section{Statistical Analysis}

Power calculations were performed using R v. 3.1.2 (18). On the basis of the effects reported in genome-wide association studies and with the MAFs available, the statistical power value needed to detect a significant association ( $P<0.016$; i.e., 0.05/3 SNPs) with dietary fibre intake $\log$ (fiber) $\sim$ GSTA+GSTT+GSTM in our study was $13 \%$. When considering women $(n=350)$ and men $(n=$ 122) separately, the power increased to $72 \%$, and $85 \%$, respectively.

The basic descriptive statistics of continuous variables were represented by mean $\pm s d$. Distribution of categorical variables was expressed in absolute frequencies. Independence among categorical variables was tested using Pearson's $\chi^{2}$ test. The Hardy-Weinberg equilibrium was tested for GSTA polymorphisms by calculating the $\chi^{2}$ test separately for obese and non-obese. The normal distribution of continuous variables was tested using statistical tests (Shapiro-Wilk, Pearson, Anderson-Darling) and graphical tools (histogram, Q-Q graph). In case of a significant violation of the normal distribution of variables, logarithmic and square-root transformation was performed and normality was tested again. To identify differences between the distributions of a continuous variable in independent groups, parametric (t-test, Welch test, ANOVA) or nonparametric (Mann-Whitney, KolmogorovSmirnov, Kruskal-Wallis) tests were performed. Linear regression was used for modeling total energy intake, fiber, body fat and waist circumference with sex, age, BMI and GSTs as independent variables. Vitamin $\mathrm{C}$ was modeled using generalized linear regression for gamma distribution with the same set of independent variables as for waist circumference. These models were subsequently reduced using backward stepwise regression based on the Akaike information criterion (AIC). Values of $p<0.05$ were considered statistically significant. Benjamini-Hochberg correction to multiple comparisons was used, where appropriate and only the corrected values are reported. All calculations were performed using statistical software R, v. 3.1.2.

\section{Results}

\section{Descriptive statistics}

The cohort description is provided in Table 1. Full anthropometric and nutritional data were obtained from a group of 472 subjects, which included 350 men and 122 women with a mean age of $45.26 \pm 13.26$ years, mean BMI of $32.36 \pm 7.71 \mathrm{~kg} / \mathrm{m}^{2}$, mean body fat percentage of $36.79 \pm 10.88 \%$ and mean waist circumference of $100.94 \pm 18.82 \mathrm{~cm}$. Mean total energy intake was 8,682 $\pm 2,738 \mathrm{~kJ}$ per day, fiber intake $15.69 \pm 5.89 \mathrm{~g}$ per day and vitamin C intake $95.34 \pm 57.55 \mathrm{mg}$ per day. The investigated classes of human GSTs were class Alpha (GSTA), Mu (GSTM) and Theta (GSTT), the selected polymorphisms were SNPs in GSTA1 (C69T substitution) and deletion in GSTM1 and GSTT1 genes. The investigated SNPs were tested for the Hardy-Weinberg equilibrium and the polymorphisms were 
found to be independent of one another and also independent of gender. The GSTA genotype distribution was 160, 230 and 82 for the CC, AC and AA genotype respectively, the GSTT was 96 and 376 for the genotype with deletion and the wild type and the GSTM genotype distribution was 230 and 242 for the genotype with deletion and the wild type.

\section{Association of investigated polymorphisms with anthropometric parameters}

A linear regression model was employed to find the association between studied polymorphisms and anthropometric parameters such as BMI, body fat and waist circumference. Models using anthropometric parameters as dependent variables and genotypes of the investigated polymorphisms, sex, age and total energy intake as independent variables were subsequently developed. Age was found to be a significant predicting factor for $\mathrm{BMI}$, showing the relation of higher age and higher $\mathrm{BMI}(\beta=0.02,95 \% \mathrm{Cl}: 0.01 ; 0.03, \mathrm{p}<0.001)$ with squared age as also significant $(\beta=-$ $0.0002,95 \% \mathrm{Cl}:-0.0003 ;-0.0001, p=0.004)$. No statistically significant differences within groups with different allelic variants were established. No correlations with independent variables were found in the case of body fat percentage, apart from its expected relationship with BMI, age and sex. The results of the waist circumference model confirmed our previous findings, i.e. no relationship with the GST polymorphisms was established.

\section{Association of investigated polymorphisms with nutritional parameters}

A linear regression model was used to demonstrate the correlation of polymorphisms with dietary composition. Total energy intake, vitamin $\mathrm{C}$ intake and fiber intake were used as dependent variables, while the polymorphisms, sex, BMI and age were used as independent variables. None of the allelic variants served as an independent predictor of total energy intake. The only variable with a statistically significant correlation with daily energy intake was age $(\beta=-0.003,95 \% \mathrm{Cl}:-0.006 ;-0.001$, $p=0.007)$, demonstrating the relationship between higher age and decreased nutrition. The model for vitamin $\mathrm{C}$ showed no significant correlation with the above mentioned independent variables such as individual polymorphisms, gender, BMI and age. However, statistically significant differences were observed in the case of daily fiber intake. Correlation was found for the GSTA1 and GSTM1 genes and the final model was thus created using the following symbolic regression model formula:

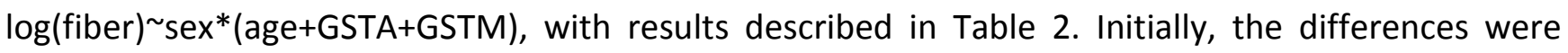
investigated for men and women separately. Men carrying the AC heterozygous genotype of GSTA1 were found to exhibit significantly increased fiber intake compared to AA homozygous men ( $p=$ $0.005)$. Men with the GSTM1 wild type gene had significantly increased fiber intake compared to men with GSTM1 deletion $(p=0.032)$. On the other hand, women with the GSTM1 wild type had significantly decreased fiber intake compared to women with GSTM1 deletion ( $p=0.022)$. When the sub-cohorts of both sexes were compared, statistically significant differences were found between AA homozygous men and AA homozygous women in GSTA1, between AA homozygous men and $A C$ heterozygous women in GSTA1 and between AC heterozygous men and AA homozygous women in GSTA1. In the case of the GSTM1 gene, statistically significant differences in fiber intake were found when comparing men with deletion and women with the wild type gene and when comparing men with the wild type gene and women with deletion. All p-values are included in Table 3 and Table 4. Investigated polymorphisms in GSTT1 genes failed to show a statistically significant association with daily fiber intake. 


\section{Discussion}

The importance of metabolic phenotypes in nutrition has been previously discussed; however, the results have not been conclusive. The role of genetics in the human metabolism is unquestionable, though variability in metabolic enzyme activity is only one of the contributing aspects. Digestion, absorption and the entire metabolism of specific dietary compounds is a complex process involving different metabolic pathways. This study focuses on compounds metabolized by glutathione conjugation, which - according to previous studies - include primarily phytochemicals and are therefore found in fruits and vegetables.

Frequently studied phytochemicals detoxified by GST are isothiocyanates derived from glucosinolates found especially in brassica vegetables. These compounds were observed to differ in their cytotoxicity (19), which is compound-specific and generally associated with chemopreventive properties. However, while some studies mention the role of GST in the metabolism of other food compounds, e.g. heterocyclic amines found in cooked meat (20), no studies dealing with the specific toxicity of brassica vegetables or other vegetables rich in compounds metabolized through the GST pathway in humans are currently available and a further investigation is thus needed. However, a previous study on kinetics of sulforpahane, an isothiocyanate derived from the 4-methylsulfinylbutyl glucosinolate that accumulates in broccoli florets, suggests that lack of GSTM1 enzyme could be associated with faster excretion of sulforaphane in subjects eating broccoli (21) and the authors suggest that GSTM1 genotypes have a significant effect on the metabolism of sulforaphane derived from either standard or high-glucosinolate broccoli. Based on these observations, it can be suggested that a proportion of sulforaphane may be retained within the body, rather than rapidly excreted and the authors also speculate that such retention could possibly affect food preferences for brassica vegetables. The questions how genetic variability in GSTs affects food preferences for selected food groups, mainly brassica vegetables, however, remains still under investigation.

Using anthropometric models, no correlation was found between GST polymorphisms and BMI, total body fat and waist circumference. Since no link between the selected polymorphisms and obesity was discovered, the nutritional data of the whole sample can be considered representative even though the BMI was above average. Furthermore, our results suggest that the dietary preferences influenced by the studied polymorphisms do not alter dietary structure significantly enough to affect elementary macronutrient intake and body composition. The nutritional models did not show any correlation between vitamin $C$ intake and the investigated polymorphisms. However, vitamin $C$ intake in the studied group was generally higher than the adequate daily intake. Nowadays, vitamin $\mathrm{C}$ deficiency is very uncommon in developed countries. Taking into consideration the modern-day diet, the differences in the intake of some vegetable species are not very likely to influence vitamin $C$ intake as such. By contrast, the average fiber intake in the studied group was below the recommended daily amount and was therefore found to serve as a better indicator of food preference variability. Statistically significant differences were found for polymorphisms in GSTA1 and GSTM1, though the GSTM1 results were contradictory in men and women. It is important to consider that the number of men in the studied group was significantly lower and possibly cannot serve as a representative sample. Furthermore, the validity and reliability of self-reported data could differ by gender. No statistically significant correlation with total energy intake was found, which corresponds to our 
anthropometric models.

The major strength of this study is the size and structure of the cohort, which included a wide age range of individuals from a homogenous white Central European population. All food records were based on a self-administered 7-day questionnaire, as opposed to a standard 3-day questionnaire used in other designs, to provide more accurate data. The subjects were interviewed twice by a nutritional specialist in order to account for reporting bias. None of the individuals were following any specific diet. All models were adjusted for basic sociodemographic parameters.

This study was generally based on the hypothesis that the described genetic variability could affect the food choices by the speed of metabolization of sulforaphane and similar substances and that the time of persistence of isothiocyanates in the body affects food preferences/aversions of the individual. A part of working hypothesis was that certain aversions could result in the insufficient intake of important nutrients and lower or higher total energy intake and thus to different anthropometric parameters. However, our study did not utilize direct measurements of food preferences/aversions and thus the conclusions can be considered only preliminary and require further validation. Moreover, adjustment for all variables contributing to obesity is impossible; therefore, it is likely that some factors were not accounted for. The mean age in the cohort was 45.26 years which could introduce age-dependent bias with respect to BMI and body fat.. The number of women in the sample was significantly higher than the number of men, which must be taken into consideration when interpreting models for separate gender sub-cohorts. However, as the results of the power analysis suggest the power to detect the observed association with dietary fiber was sufficient when considering both males and females, separately. Furthermore, while the validity of the reported data may be decreasing towards negatively skewed BMI distribution, this is a standard limitation of studies with self-reported data. Additionally, while our study uses fiber and vitamin $\mathrm{C}$ to represent the amount of consumed fruit and vegetables, it cannot be considered fully representative of any selected phytochemical intake nor isothiocyanates intake.

Although the results do not show a clear dependency of any polymorphism on either the higher or lower intake of both components associated with vegetable intake, a significant relationship with dietary fibre is apparent in some of the individual models. The results therefore confirm, at least partially, our hypothesis that some GST polymorphisms may be associated with different food choices while still taking into consideration that the actual food composition may not always fully reflect food preferences. Supposing that individuals with studied GST polymorphisms are slow metabolizers of certain phytochemicals, higher metabolite plasma concentrations may be causing specific food intolerances or aversions. If this association with genetics were confirmed, introducing an individualized diet would be reasonable in order to substitute some foods while still meeting nutrient recommendations.

To conclude, this study shows a significant relationship of unmodified, native daily fiber intake with selected GSTA1 and GSTM1 polymorphisms in both men and women. There is, however, a need for further studies using a cohort with the standard distribution of age and sex and using more precise nutritional methodology (possibly investigating specifically food preferences/aversions). Taking into account other compounds metabolized through GST, an analysis of specific food groups is necessary in follow-up research. However, we believe that the observed differences in daily fiber intake suggest 
that the genetic variability of glutathione S-Transferase may play a role in dietary preferences and may therefore lead to specific nutrient deficiencies.

\section{Statements}

\section{Ethics approval and consent to participate}

The present study was conducted according to Declaration of Helsinki guidelines and all procedures involving human subjects were approved by the Committee for Ethics of Medical Experiments on Human Subjects, Faculty of Medicine, Masaryk University, Brno, Czech Republic.

\section{Competing interests}

The authors declare that they have no competing interests.

\section{Funding}

This research was financially supported by Masaryk University (CETOCOEN PLUS; CZ.02.1.01/0.0/0.0/15_003/0000469) and the RECETOX Research Infrastructure (LM2015051 project of the Ministry of Education, Youth and Sports of the Czech Republic).

\section{Authors' contributions}

JBV designed the study and provided critical review. MF analyzed the food records. FZ performed the statistical analysis. BK wrote the manuscript and has the primary responsibility for the content. HP critically revised the manuscript. All of the authors read and approved the final manuscript.

\section{Acknowledgements}

The authors are grateful to all study participants and to David Konečný for writing assistance, language editing and proofreading. 


\section{References}

1. Anonymous. Diet, nutrition and the prevention of chronic diseases. .World Health Organ. Tech. Rep. Ser.; 2003;916:i-viii, 1-149, backcover.

2. McGuire S. U.S. Department of Agriculture and U.S. Department of Health and Human Services, Dietary Guidelines for Americans, 2010. 7th Edition, Washington, DC: U.S. Government Printing Office, January 2011. .Adv. Nutr.; 2011;2:293-294.

3. Anonymous. Vitamin and mineral requirements in human nutrition [report of a joint FAO/WHO expert consultation, Bangkok, Thailand, 21-30 September 1998 . 2nd ed. Geneva : World Health Organization, 2004.

4. The European Food Safety Society: The EFSA Comprehensive European Food Consumption Database, accessed March 2017.

5. Holmes E, Loo RL, Stamler J, et al. Human metabolic phenotype diversity and its association with diet and blood pressure. .Nature.; 2008;453:396-400.

6. Rezzi S, Ramadan Z, Martin F-PJ, et al. Human metabolic phenotypes link directly to specific dietary preferences in healthy individuals. .J. Proteome Res.; 2007;6:4469-4477.

7. Sheehan D, Meade G, Foley VM, Dowd CA. Structure, function and evolution of glutathione transferases: implications for classification of non-mammalian members of an ancient enzyme superfamily. .Biochem. J.; 2001;360:1-16.

8. Lampe JW. Diet, genetic polymorphisms, detoxification, and health risks. .Altern. Ther. Health Med.; 2007;13:S108-111.

9. Conaway CC, Yang Y-M, Chung F-L. Isothiocyanates as cancer chemopreventive agents: their biological activities and metabolism in rodents and humans. .Curr. Drug Metab.; 2002;3:233-255.

10. Yang Y, Yan H, Li Y, et al. Isothiocyanates from Broccolini seeds induce apoptosis in human colon cancer cells: proteomic and bioinformatic analyses. .Pharm.; 2011;66:382-390.

11. Shapiro TA, Fahey JW, Wade KL, et al. Human metabolism and excretion of cancer chemoprotective glucosinolates and isothiocyanates of cruciferous vegetables. .Cancer Epidemiol. Biomark. Prev. Publ. Am. Assoc. Cancer Res. Cosponsored Am. Soc. Prev. Oncol.; 1998;7:1091-1100.

12. Bienertova-Vasku J, Bienert $P$, Tomandl J, et al. No association of defined variability in leptin, leptin receptor, adiponectin, proopiomelanocortin and ghrelin gene with food preferences in the Czech population. .Nutr. Neurosci.; 2008;11:2-8.

13. Bienertová-Vasků J, Bienert $\mathrm{P}$, Forejt $\mathrm{M}$, et al. Genotype x nutrient association of common polymorphisms in obesity-related genes with food preferences and time structure of energy intake. .Br. J. Nutr.; 2010;103:352-359.

14. Hezova R, Bienertova-Vasku J, Sachlova M, et al. Common polymorphisms in GSTM1, GSTT1, GSTP1, GSTA1 and susceptibility to colorectal cancer in the Central European population. .Eur. J. Med. Res.; 2012;17:17. 
15. Ping J, Wang H, Huang M, Liu Z-S. Genetic analysis of glutathione S-transferase A1 polymorphism in the Chinese population and the influence of genotype on enzymatic properties. .Toxicol. Sci. Off. J. Soc. Toxicol.; 2006;89:438-443.

16. Chen CL, Liu Q, Relling MV. Simultaneous characterization of glutathione S-transferase M1 and T1 polymorphisms by polymerase chain reaction in American whites and blacks. .Pharmacogenetics.; 1996;6:187-191.

17. Naveen AT, Adithan C, Padmaja N, et al. Glutathione S-transferase M1 and T1 null genotype distribution in South Indians. .Eur. J. Clin. Pharmacol.; 2004;60:403-406.

18. R Core Team. R: A language and environment for statistical computing. R Foundation for Statistical Computing, Vienna, Austria, 2013. Available from http://www.R-project.org/.

19. Kadir NHA, David R, Rossiter JT, Gooderham NJ. The selective cytotoxicity of the alkenyl glucosinolate hydrolysis products and their presence in Brassica vegetables. .Toxicology.; 2015;334:59-71.

20. Van Hemelrijck M, Rohrmann S, Steinbrecher A, et al. Heterocyclic aromatic amine [HCA] intake and prostate cancer risk: effect modification by genetic variants. .Nutr. Cancer.; 2012;64:704-713.

21. Gasper AV, Al-janobi A, Smith JA, et al. Glutathione S-transferase M1 polymorphism and metabolism of sulforaphane from standard and high-glucosinolate broccoli. .Am. J. Clin. Nutr.; 2005;82:1283-1291. 
Table 1. Description of the study subjects

\begin{tabular}{|l|r|r|r|r|r|r|r|}
\hline & $\mathrm{n}$ & Mean & $\mathrm{SD}$ & Median & Min & Max & Range \\
\hline Height (cm) & 472 & 167.72 & 9.03 & 167 & 146.5 & 197 & 50.5 \\
\hline Weight (kg) & 472 & 91.04 & 22.8 & 89.55 & 43.5 & 183.3 & 139.8 \\
\hline BMI (kg/m2) & 472 & 32.36 & 7.71 & 31.77 & 15.76 & 63.13 & 47.36 \\
\hline Age (years) & 472 & 45.26 & 13.26 & 46.75 & 15.8 & 73.2 & 57.4 \\
\hline $\begin{array}{l}\text { Systolic blood pressure } \\
\text { (mmHg) }\end{array}$ & 470 & 132.07 & 19.73 & 130 & 87 & 203 & 116 \\
\hline $\begin{array}{l}\text { Diastolic blood pressure } \\
\text { (mmHg) }\end{array}$ & 470 & 86.97 & 12.57 & 86 & 54 & 134 & 80 \\
\hline $\begin{array}{l}\text { Skinfold thickness summary } \\
\text { (cm) }\end{array}$ & 457 & 91.72 & 29.39 & 91 & 30 & 191 & 161 \\
\hline Body fat (\%) & 472 & 36.79 & 10.88 & 37.55 & 7.1 & 63.8 & 56.7 \\
\hline Waist circumference (cm) & 472 & 100.94 & 18.82 & 100 & 58 & 162 & 104 \\
\hline Waist : hip ratio & 472 & 0.88 & 0.1 & 0.88 & 0.67 & 1.16 & 0.49 \\
\hline $\begin{array}{l}\text { Total daily energy intake } \\
\text { (kJ/day) }\end{array}$ & 472 & 8681.59 & 2737.59 & 8327 & 2278 & 21,591 & 19,313 \\
\hline Daily fiber intake (g/day) & 472 & 15.69 & 5.89 & 15.14 & 4 & 53.7 & 49.7 \\
\hline $\begin{array}{l}\text { Daily vitamin C intake } \\
\text { (mg/day) }\end{array}$ & 472 & 95.34 & 57.55 & 85.75 & 11.23 & 390.14 & 378.91 \\
\hline
\end{tabular}


Table 2. Final daily fiber intake model output

\begin{tabular}{|c|c|c|c|c|}
\hline $\begin{array}{l}\text { Independent } \\
\text { variable }\end{array}$ & $\begin{array}{l}\text { Difference between } \\
\text { levels }\end{array}$ & $\beta$ Estimate & 95\% Wald Cl & $p$-Value \\
\hline \multirow{2}{*}{ Sex } & Female & \multicolumn{3}{|c|}{ Ref } \\
\hline & Male & -0.209 & $(-0.484 ; 0.067)$ & 0.139 \\
\hline Age-females & - & 0.001 & $(-0.002 ; 0.004)$ & 0.401 \\
\hline Age-males & - & 0.006 & $(0.001 ; 0.011)$ & 0.011 \\
\hline GSTA-females & $A C-C C$ & -0.024 & $(-0.10 ; 0.06)$ & 0.552 \\
\hline \multirow{5}{*}{ GSTA-males } & $\mathrm{AA}-\mathrm{CC}$ & 0.072 & $(-0.04 ; 0.18)$ & 0.201 \\
\hline & $A A-A C$ & 0.096 & $(-0.01 ; 0.20)$ & 0.073 \\
\hline & AC-CC & 0.132 & $(-0.01 ; 0.27)$ & 0.064 \\
\hline & AA-CC & -0.097 & $(-0.27 ; 0.07)$ & 0.267 \\
\hline & $A A-A C$ & -0.229 & $(-0.39 ;-0.07)$ & 0.005 \\
\hline GSTM-females & Wild type-deletion & -0.085 & $(-0.16 ;-0.01)$ & 0.022 \\
\hline GSTM-males & Wild type-deletion & 0.136 & $(0.01 ; 0.26)$ & 0.032 \\
\hline \multirow{2}{*}{ Sex : age } & Female & \multicolumn{3}{|c|}{ Ref } \\
\hline & Male & 0.005 & $(-0.005 ; 0.010)$ & 0.076 \\
\hline \multirow{3}{*}{ Sex : GSTA } & Female : CC & \multicolumn{3}{|c|}{ Ref } \\
\hline & Male : AC & 0.157 & $(-0.004 ; 0.318)$ & 0.057 \\
\hline & Male : AA & -0.168 & $(-0.371 ; 0.034)$ & 0.104 \\
\hline \multirow{2}{*}{ Sex : GSTM } & Female : deletion & \multicolumn{3}{|c|}{ Ref } \\
\hline & Male : wild type & 0.222 & $(0.078 ; 0.366)$ & 0.003 \\
\hline
\end{tabular}

Cl confidence interval, GST glutathione S-transferases. 
Table 3. GSTA1 p-values: subcohort comparison

\begin{tabular}{|l|r|r|r|r|l|}
\hline & Women CC & Women AC & Women AA & Men CC & Men AC \\
\hline Women AC & 0.552 & & & & \\
\hline Women AA & 0.201 & 0.073 & & & \\
\hline Men CC & 0.139 & 0.057 & 0.104 & & \\
\hline Men AC & 0.057 & 0.697 & $\mathbf{0 . 0 0 1}$ & 0.064 & \\
\hline Men AA & 0.104 & $\mathbf{0 . 0 0 1}$ & $\mathbf{0 . 0 1 1}$ & 0.267 & $\mathbf{0 . 0 0 5}$ \\
\hline
\end{tabular}

Table 4. GSTM1 p-values: subcohort comparison

\begin{tabular}{|l|r|r|r|}
\hline & Women deletion & Women wild type & Men deletion \\
\hline Women wild type & $\mathbf{0 . 0 2 2}$ & & \\
\hline Men deletion & 0.139 & $\mathbf{0 . 0 0 3}$ & \\
\hline Men wild type & $\mathbf{0 . 0 0 3}$ & 0.93 & $\mathbf{0 . 0 3 2}$ \\
\hline
\end{tabular}

